\section{Errors in Chromatography}

R. Kaiser

BASF, D-67 Ludwigshafen/Rh.
Die gleiche Arbeit können Sie in deutscher Sprache auf Seite 126 lesen

Vous trouverez ce travail en langue française en page 129
The following series of the Green Pages deals with the random error and the systematic error in chromatographic analysis and in the evaluation of the results. Only the simplest mathematical relations are being employed, which just permit to make statistic-mathematical assertions.

Errors due to instrumentation and application, including the sampling error and methods for its discovery, will be discussed in a later issue.

\section{Part I. Random error}

"Random errors are due to fluctuations in the sources of error which cannot be determined, and which are independent from the will of the observer. They are unavoidable".

This is a definition of the random error. In other words: By the law of the nature, each measurement is subject to errors, the extent and direction of which fluctuate statistically. Although the systematic error will be discussed only in a later issue of the Green Pages, we should state already here, that in practical work it is not possible to make a sharp distinction between true statistical random errors and a true systematic error. Fluctuating systematic errors are of ten being considered as random errors. In chromatographic analysis the systematic error often exceeds the random error by more than one order of magnitude. Nevertheless let us agree here on the following:

The data groups discussed hereafter do not include any systematic error. We are going to call the results of measurements as "data". It is unimportant whether these data represent the qualitative composition of a sample, the concentration in volume percent, weight percent, etc., or whether they are $\mathrm{Rf}$ values, retention volumes, diffusion coefficients, or polarity values.

In order to be able to make a judgment or a decision on the basis of data, the following conditions must be met:

1. The data must be free from systematic errors or must permit the search for systematic errors, or it must be certain that systematic errors do not influence the judgment or the decision.

2. The data must represent mean values from several measurements. Mean value $=\overline{\mathbf{x}}$.

3. The standard deviation of the data must be known. Standard deviation $=\mathrm{s}$.
4. The number of repetitive or comparative measurements used for calculating the mean value must be known. Number of measurements $=n$.

Without knowing the three characteristic values $\bar{x}, s$, and $\mathrm{n}$ it is never possible to make a judgment or a decision on the basis of data which are scientifically reliable.

Each mathematical-statistical assertion can be made only with a limited certainty; we call it the statistical certainty, $\mathrm{S}$ and it is given in \%.

Internationally the following standards are beginning to be adopted: A statistical certainty of $95 \%$ is chosen for all decisions concerning the evaluation of a product.

$\mathrm{S}=99 \%$ is chosen when scientific conclusions have to be drawn.

$\mathrm{S}=99.9 \%$ is chosen when the decisions entail high in vestments or concern large material values.

The rigorousness of all statistical tests and results depends on the chosen statistical certainty.

\section{Runaway values, mean value, standard deviation}

It is the nature of probability that excessive errors may appear in each measurement. At least three repetitive measurements are necessary before one of the measurements may be judged as extremely incorrect. The more repetitive measurements are available, the better it is possible to recognize runaway values.

Runaway values must be eliminated before the data may be used for any decisions

For our purposes, the best test for runaway values is the NALIMOV Test. It has advantages over numerous other tests for runaway values.

\section{NALIMOV Test}

Before it can be employed, it is necessary to calculate from all data, including the runaway values a preliminary mean value $\bar{x}$ and a preliminary standard deviation $s$.

1. Calculate the mean value $\bar{x}$ of all individual values

$$
\text { Formula: } \begin{aligned}
& \bar{x}=\left(x_{1}+x_{2}+x_{3}+\ldots\right) / n \\
& n=\text { number of all individual measurements } \\
& \text { Example: } \\
& x_{1}=30.41 \\
& x_{2}=30.05 \\
& x_{3}=30.49 \\
& x_{4}=32.06 \\
& \bar{x}=123.01 / 4 \\
& \bar{x}=30.753
\end{aligned}
$$




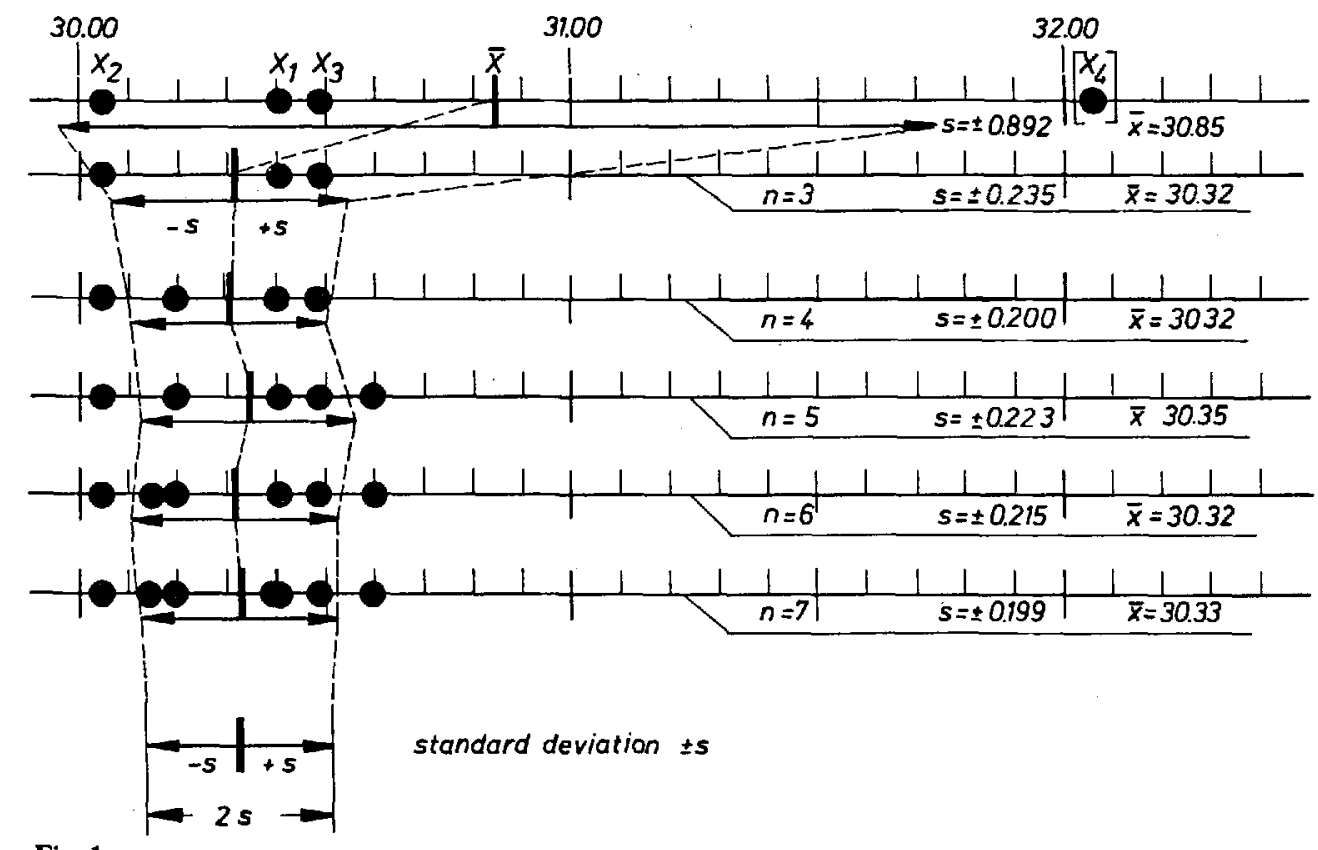

Fig 1

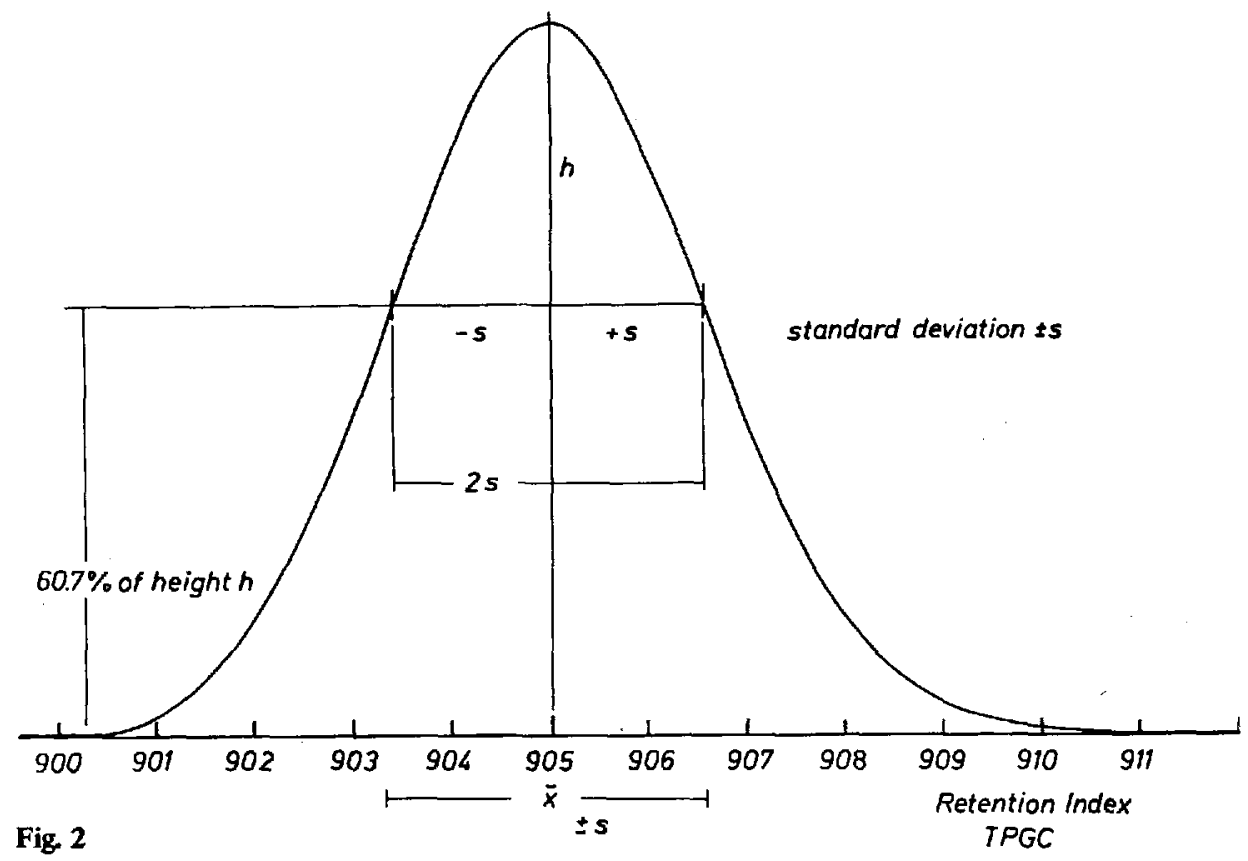

2. Calculate the preliminary standard deviation $s$ of all individual values

Example:

$s= \pm \sqrt{\frac{1}{n-1} \cdot\left[\Sigma\left(x^{2}\right)-\frac{1}{n}(\Sigma x)^{2}\right]}$

$\mathrm{n}=$ number of individual measurements $x_{1}=30.41$

$\mathrm{x}_{2}=30.05$

$\mathrm{x}_{3}=30.49$

$\mathrm{x}_{4}=32.06$

$\Sigma \mathrm{x}=123.01 \quad \Sigma\left(\mathrm{x}^{2}\right)=3785.2543$

$\mathrm{x}^{2}$

924.7681

903.0025

929.6401

1027.8436

$(\Sigma x)^{2}=15131.4601$

$$
\begin{aligned}
& s= \pm \sqrt{\frac{1}{3}\left(3785.2543-\frac{1}{4} \cdot 15131.4601\right)} \\
& s= \pm 0.8924
\end{aligned}
$$




\section{Remark:}

When using a calculator, the full number of digits of the, intermediary result must be kept. Only the final result can be reduced to the number of digits which corresponds to the accuracy of the measurement.

\section{Test for nunaway values}

Check the data for any runaway values. The highest and the lowest measuring value(s) must be suspected.

For this purpose, compare the suspected runaway values with a value obtained by means of test formula (1), using from Table 1 the n-dependent Nalimov factor N(95), $\mathrm{N}(99)$, or $\mathrm{N}(99.9)$.

\section{Test formula 1}

$$
\begin{aligned}
& x^{*} \quad \text { is not a runaway value if } \\
& \left|x^{*}-\bar{x}\right| \text { is lower than } N(95) \cdot s \\
& x^{*} \quad \text { is probably a runaway value if } \\
& \left|x^{*}-\bar{x}\right| \text { is equal to or higher than } N(95) \cdot s \\
& x^{*} \quad \text { is a significant runaway value if } \\
& \left|x^{*}-\bar{x}\right| \text { is equal to or higher than } N(99) \cdot s \\
& x^{*} \quad \text { is a most significant nunaway value if } \\
& \left|x^{*}-\bar{x}\right| \text { is equal to or higher than } N(99.9) \cdot s
\end{aligned}
$$

\section{Application:}

It follows from the earlier numerical example that: (see Table 1)

$\mathrm{N}(95) \cdot \mathrm{s}=1.425 \cdot 0.8924=1.2717$

$\mathrm{N}(99) \cdot \mathrm{s}=1.485 \cdot 0.8924=1.3252$

$\mathrm{N}(99.9) \cdot \mathrm{s}=1.498 \cdot 0.8924=1.3368$

$\mathrm{x}_{1}=\mathrm{x}^{*}=32.06 ;\left|\mathrm{x}^{*}-\overline{\mathrm{x}}\right|=32.06-30.753=\underline{1.3070}$

$x_{1}, x_{2}$, and $x_{3}$ would give a much smaller difference.

The consequence is: Only $x_{4}=32.06$ is, with $95 \%$ probability, a runaway value. It must be eliminated.

The data are now free from runaway values.

Now calculate the final characteristic data for $\overline{\mathrm{x}}, \mathrm{s}$, and $\mathrm{n}$ :

$$
\begin{aligned}
& \mathrm{n}=\mathbf{3} \\
& \left.\overline{\mathrm{x}}_{\mathrm{A}}=30.317 \quad \text { (because }(30.41+30.05+30.49) / 3=30.317\right) \\
& \mathrm{s}_{\mathrm{A}}= \pm 0.2345 \text { (because } \sqrt{\frac{1}{2}\left(2757.4107-\frac{1}{3} \cdot 8271.9025\right)} \\
& = \pm 0.2345 \text { ) }
\end{aligned}
$$

Only now is it possible to make a judgment based on mathematical facts:

Does the measuring result $\vec{x}_{A}$ indeed differ from another one $\bar{x}_{B}$ or from a nominal value $W$ ? With regard to the quality of measurement, does the used measuring procedure which produced the standard deviation $\mathrm{s}_{\mathrm{A}}$ indeed differ from another measuring procedure which produced values with a standard deviation $\mathrm{s}_{\mathrm{B}}$ ?

These questions, a highly sensitive test for differences in the mean values of paired measurements, and a simple graphic method of statistical mathematics, will be treated in the next issue of the Green Pages.

The methods of calculation discussed here and in later issues require a statistical "normal distribution" of the data. A test regarding this question will also be dealt with in the next issue.

Finally, the significance of the mean value $\bar{x}$ and of the standard deviation $s$ are explained here by means of the figures 1 and 2:

Fig. 1 shows a graduated scale of values. The four numerical values of our example have been entered in this scale. It can be recognized how far the range of the standard deviation exceeds the values which are higher and lower than the mean value.

If more than four repetitive measurements would have been made - from which one would have to be eliminated as a runaway value -, then the mean value would have been different and, the standard deviation would have been smaller al though only slightly. In the other graduated scales of Fig. 1, the changes in the data which may then be expected have been entered.

In Fig. 2 we have applied the terms "mean value" and "standard deviation" to the retention time of a gas chromatographic peak. When calculating the retention index of a peak, the resulting value should actually be given only together with the standard deviation resulting from the shape of the peak. For this particular example we assume that the analysis had been carried out at programmed temperature, resulting in a linear retention index scale. 


\section{Fehler in der Chromatographie}

\section{R. Kaiser}

BASF, D-67 Ludwigshafen/Rh.
You can read this paper in English language on page 123

Vous trouverez ce travail en langue française en page 129
Die folgende Serie der grünen Seiten beschäftigt sich mit dem Zufallsfehler und dem systematischen Fehler bei chromatographischen Analysen und bei der Auswertung der Ergebnisse. Es werden nur die einfachsten rechnerischen Beziehungen angewendet, welche gerade noch statistisch-mathematische Aussagen zulassen.

Instrumentelle und anwendungstechnische Fehler, einschließlich des Probennahmefehlers und Methoden zu dessen Entdeckung werden ebenfalls in einer späteren Folge diskutiert.

\section{Teil I: Zufallsfehler}

„Zufallsfehler rühren von nicht bestimmbaren, vom Willen des Beobachters unabhängigen Schwankungen in den Fehlerquellen her. Sie sind unvermeidbar."

Dies ist eine Definition des Zufallsfehlers. Man kann auch sagen: Jede Messung ist naturgesetzlich mit Fehlern behaftet, deren Größe und Richtung statistisch schwankt. Obwohl der systematische Fehler erst in einer späteren Folge der grünen Seiten diskutiert wird, sei schon hier festgestellt:

Eine scharfe Trennung zwischen den echten statistischen Zufallsfehlern und einem echten systematischen Fehler ist in der Praxis nicht möglich. Oft werden schwankende systematische Fehler als Zufallsfehler behandelt. In der chromatographischen Analyse ist der systematische Fehler häufig um mehr als eine Größenordnung größer als der Zufallsfehler. Trotzdem wollen wir hier vereinbaren:

Die im folgenden behandelten Meßwertegruppen sind frei von systematischen Fehlern. Wir wollen die Meßergebnisse „Daten" nennen. Es ist dabei gleichgiltig, ob die Daten die qualitative Zusammensetzung einer Probe, den Gehalt in Vol.-\%, Gew.-\% usw. oder ob sie Rf-Werte, Retentionsvolumina, Retentionsindizes, Diffusionskoeffizienten oder Polaritätswerte wiedergeben.

Um aus Daten ein Urteil oder eine Entscheidung fällen zu könnèn, müssen folgende Bedingungen erfüllt sein:

1. Die Daten müssen frei von systematischen Fehlern sein oder zur Suche nach systematischen Fehlern dienen, oder es muß sicher sein, daß systematische Fehler das Urteil bzw. die Entscheidung nicht beeinflussen.

2. Die Daten müssen Mittelwerte aus mehreren Messungen sein. Mittelwert $=\overline{\mathbf{x}}$.

3. Die Standardabweichung der Daten muß bekannt sein. Standardabweichung $=\mathbf{s}$.
4. Die Zahl der Wiederhol- oder Vergleichsmessungen, aus welchen der Mittelwert berechnet wurde, muß bekannt sein. Zahl der Messungen $=n$.

Ohne Kenntnis der drei Kenngrößen $\bar{x}$, s und n kann nie ein Urteil oder eine Entscheidung aus Daten gefällt werden, das naturwissenschaftlich einwandfrei ist.

Jede mathematisch-statistische Aussage hat nur eine begrenzte Sicherheit, wir bezeichnen sie als statistische Sicherheit $S$ in $\%$.

International beginnt sich durchzusetzen:

$\mathrm{S}=95 \%$ wird für alle Produktbeurteilungen gewählt.

$\mathrm{S}=99 \%$ wird gewählt, wenn wissenschaftliche Schlußfolgerungen gezogen werden.

$S=99,9 \%$ wird gewählt, wenn die Entscheidungen zu hohen Investitionen führen oder sonst große materielle Werte betreffen.

Die Schärfe aller statistischer Tests und Ergebnisse richtet sich nach der gewählten statistischen Sicherheit.

\section{Ausreißer, Mittelwert, Standardabweichung}

Es liegt in der Natur des Zufalls, daß übergroße Fehler bei jeder Messung auftreten können. Man benötigt mindestens drei Wieđerholmessungen, ehe beurteilt werden kann, ob eine Messung davon extrem falsch ist. Je mehr Wiederholungen vorliegen, um so schärfer kann man Ausreißer erkennen.

Ausreißer müssen entfernt werden, ehe die Daten für Entscheidungen verwendet werden diurfen.

Der für uns beste Ausreißertest ist der NALIMOV-Test. Er hat Vorteile vor zahlreichen anderen Ausreißertests.

\section{NALIMOV-Test}

$\mathrm{Zu}$ seiner Anwendung muß aus allen Daten, einschließlich Ausreißern, ein vorläufiger Mittelwert $\bar{x}$ und eine vorläufige Standardabweichung s berechnet werden.

\section{Berechne den Mittelwert $\overline{\mathrm{x}}$ aus allen Einzelwerten}

$$
\text { Beispiel: } \begin{aligned}
& \mathrm{x}_{1}=30,41 \\
& \mathrm{x}_{2}=30,05 \\
& \mathrm{x}_{3}=30,49 \\
& \mathrm{x}_{4}=32,06 \\
& \overline{\mathrm{x}}=\overline{123,01 / 4} \\
& \overline{\mathrm{x}}=30,753
\end{aligned}
$$$$
\text { Formel: } \quad \bar{x}=\left(x_{1}+x_{2}+x_{3}+\ldots\right) / n
$$$$
\mathrm{n}=\mathrm{Zahl} \text { aller Einzelmessungen }
$$ 
2. Berechne die vorläufige Standardabweichung $s$ aus allen Einzelwerten

Formel: (Diese ist besonders für die Anwendung von Tischrechenmaschinen geeigent.)

$$
s= \pm \sqrt{\frac{1}{n-1} \cdot\left[\Sigma\left(x^{2}\right)-\frac{1}{n}(\Sigma x)^{2}\right]}
$$

$\mathrm{n}=$ Zahl der Einzelmessungen

$$
\text { Beispiel: } \begin{array}{rlr} 
& x & x^{2} \\
x_{1} & =30,41 & 924,7681 \\
x_{2} & =30,05 & 903,0025 \\
x_{3} & =30,49 & 929,6401 \\
x_{4} & =32,06 & 1027,8436 \\
\Sigma x & =123,01 & \Sigma\left(x^{2}\right)=3785,2543 \\
(\Sigma x)^{2} & =15131,4601 &
\end{array}
$$

\section{Anmerkung:}

Bei Verwendung von Rechenmaschinen soll im Zwischenergebnis die volle Stellenzahl erhalten bleiben. Erst im Endergebnis soll die Zahlenangabe so weit reduziert werden, wie dies der Genauigkeit der Messung entspricht.

\section{Ausreißertest}

Prüfe das Datenmaterial auf Ausreißer. Ausreißerverdächtig sind der oder die größten und kleinsten Meßwerte.

Dazu vergleicht man die ausreißerverdächtigen Werte mit einem nach der Testformel (1) berechneten Wert, wozu man aus Tabelle 1 den von $\mathbf{n}$ abhängigen Nalimov-Faktor $\mathrm{N}(95), \mathrm{N}(99)$ oder $\mathrm{N}(99,9)$ verwendet.

\section{Testformel 1}

$$
\begin{array}{ll}
x^{*} & \begin{array}{l}
\text { ist kein Ausreißer, wenn } \\
\left|x^{*}-\bar{x}\right| \text { kleiner als } N(95) \cdot s
\end{array} \\
x^{*} \quad \begin{array}{l}
\text { ist wahrscheinlich ein Ausreißer, wenn } \\
\left|x^{*}-\bar{x}\right| \text { gleich oder größer als } N(95) \cdot s
\end{array} \\
x^{*} \quad \begin{array}{l}
\text { ist signifikant ein Ausreißer, wenn } \\
\left|x^{*}-\bar{x}\right| \text { gleich oder größer als } N(99) \cdot s
\end{array} \\
x^{*} \quad \begin{array}{l}
\text { ist hochsignifikant ein Ausreißer, wenn } \\
\left|x^{*}-\bar{x}\right| \text { gleich oder größer als } N(99,9) \cdot s
\end{array}
\end{array}
$$

\section{Anwendung:}

Aus obigem Zahlenbeispiel ergibt sich (siehe Tabelle 1):

$$
\mathrm{N}(95) \cdot \mathrm{s}=1,425 \cdot 0,8924=1,2717
$$

$\mathrm{N}(99) \cdot \mathrm{s}=1,485 \cdot 0,8924=1,3252$

$\mathrm{N}(99,9) \cdot \mathrm{s}=1,498 \cdot 0,8924=1,3368$

$\mathrm{x}_{4}=\mathrm{x}^{*}=32,06 ;\left|\mathrm{x}^{*}-\overline{\mathrm{x}}\right|=32,06-30,753=\underline{1,3070}$

$x_{1}, x_{2}$ und $x_{3}$ würden eine viel kleinere Differenz ergeben. Daraus folgt: Nur $x_{4}=32,06$ ist mit $95 \%$ iger Wahrscheinlichkeit ein Ausreißer. Er muß entfernt werden! Das Datenmaterial ist nun frei von Ausreißern. Man muß jetzt die endgültigen Kenndaten $\bar{x}$, $s$ und $n$ berechnen.
Tabelle 1. Nalimov-Faktoren

\begin{tabular}{r|l|l|l}
\hline $\mathbf{n}$ & $\mathrm{N}(95)$ & $\mathrm{N}(99)$ & $\mathrm{N}(99,9)$ \\
\hline 3 & 1,150 & 1,155 & 1,155 \\
4 & 1,425 & 1,485 & 1,498 \\
5 & 1,572 & 1,716 & 1,773 \\
6 & 1,656 & 1,872 & 1,988 \\
7 & 1,711 & 1,983 & 2,156 \\
8 & 1,749 & 2,065 & 2,289 \\
9 & 1,777 & 2,127 & 2,395 \\
10 & 1,798 & 2,176 & 2,482 \\
11 & 1,814 & 2,216 & 2,553 \\
12 & 1,829 & 2,248 & 2,614 \\
13 & 1,841 & 2,275 & 2,665 \\
14 & 1,850 & 2,298 & 2,710 \\
15 & 1,858 & 2,318 & 2,749 \\
16 & 1,865 & 2,335 & 2,783 \\
17 & 1,870 & 2,351 & 2,812 \\
18 & 1,877 & 2,363 & 2,839 \\
19 & 1,881 & 2,375 & 2,863 \\
20 & 1,886 & 2,385 & 2,884 \\
21 & 1,889 & 2,395 & 2,903 \\
22 & 1,893 & 2,403 & 2,921 \\
27 & 1,906 & 2,437 & 2,990 \\
32 & 1,914 & 2,459 & 3,036 \\
37 & 1,921 & 2,475 & 3,071 \\
42 & 1,926 & 2,488 & 3,096 \\
47 & 1,929 & 2,497 & 3,118 \\
52 & 1,932 & 2,505 & 3,135 \\
102 & 1,946 & 2,540 & 3,211 \\
502 & 1,957 & 2,567 & 3,276 \\
$\infty$ & 1,960 & 2,576 & 3,291 \\
& & &
\end{tabular}

$n=3$

$\overline{\mathrm{x}}_{\Lambda}=30,317$ (weil $(30.41+30.05+30.49) / 3=30.317$

$s_{\Lambda}= \pm 0,2345\left(\right.$ weil $\sqrt{\frac{1}{2}\left(2757.4107-\frac{1}{3} \cdot 8217.9025\right.}=$
\pm 0.2345

Erst jetzt kann ein mathematisch fundiertes Urteil gefallt werden:

Ist dieses Meßergebnis $\overline{\mathbf{x}}_{\mathbf{A}}$ wirklich unterschiedlich von einem anderen $\bar{x}_{B}$ oder von einem Sollwert $W$ ? Unterscheidet sich das verwendete Meßverfahren, das die Standardabweichung $s_{A}$ verursachte, bezüglich der Meßguite wirklich von einem anderen Meßverfahren, das Werte mit einer Standardabweichung $\mathrm{s}_{\mathbf{B}}$ verursachte?

Diese Fragen, ein hochempfindlicher Test auf Unterschiede in Mittelwerten aus paarweiser Messung und eine einfache graphische Methode der statistischen Mathematik werden in der nächsten Folge der grünen Seiten behandelt.

Die hier und später behandelten Rechenmethoden erfordern, daß das Datenmaterial statistisch ,normalverteilt" ist. Ein Test zu dieser Frage wird ebenfalls in der nächsten Folge behandelt.

Hier sei abschließend die Bedeutung des Mittelwertes $\overline{\mathbf{x}}$ und der Standardabweichung $s$ an Bild 1 und 2 erläutert:

In Bild 1 ist eine Werteskala dargestellt. Auf ihr sind die vier Zahlenwerte unseres Rechenbeispiels eingezeichnet. 

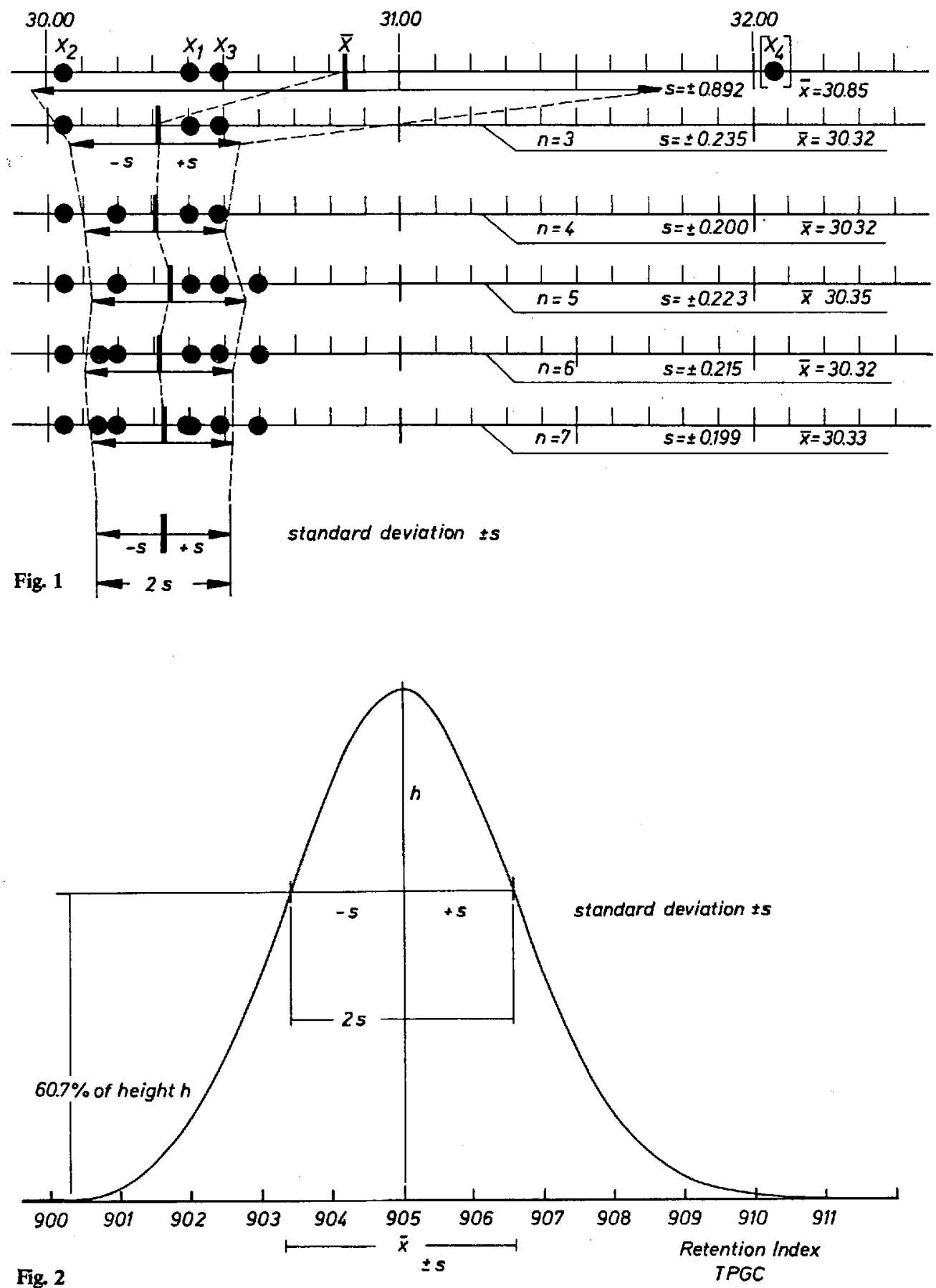

Man erkennt, wie weit der Bereich der Standardabweichung über die Werte, die größer und kleiner als der Mittelwert sind, hinausreicht.

Hätte man mehr als vier Wiederholmessungen gemacht, von denen auch noch einer als Ausreißer wegfällt, dann hätte man sowohl einen anderen Mittelwert, als auch eine (nur geringfügig) kleinere Standardabweichung gefunden. In Bild 1 sind auf den weiteren Werteskalen die dann zu erwartenden Veränderungen der Daten eingezeichnet.
In Bild 2 wenden wir die Begriffe Mittelwert und Standardabweichung auf die Retentionszeit eines gas-chromatographischen Peaks an. Rechnet man den Retentionsindex für einen Peak aus, dann dürfte man den erhaltenen Wert im Grunde genommen nur mit der Standardabwei-chung angeben, die sich aus der Form des Peaks ergibt. Es wird für dieses Beispiel angenommen, daß es sich um eine temperaturprogrammierte Analyse handelt, damit die Retentionsindexskala linear wird. 


\section{Les erreurs en Chromatographie}

\author{
R. Kaiser \\ BASF, D-67 Ludwigshafen/Rh.
}

You can read this paper in English language on page 123 Die gleiche Arbeit können Sie in deutscher Sprache auf Seite 126 lesen
La présente série de pages vertes se rapporte à l'erreur fortuite et à l'erreur systématique commises au cours des analyses chromatographiques et de l'exploitation des résultats.

On utilisera seulement les relations mathématiques les plus simples qui admettent encore des assertions statistiques exactes.

Les erreurs dues à l'appareillage et à la technique d'emploi y compris les erreurs de prélévement et les méthodes à utiliser pour les déceler feront l'objet d'une serie ultérieure.

\section{$1^{\text {ère }}$ partie - Erreurs fortuites}

Les erreurs fortuites proviennent de variations de la cause d'erreur, non déterminables et indépendantes de la volonté de l'opérateur. Elles sont inévitables.

Telle est la définition de l'erreur fortuite. Autrement dit, toute mesure est par nature entachée d'erreurs dont l'importance et le sens varient statistiquement. Bien que l'erreur systématique ne doive être étudiée que dans une prochaine série de pages vertes, nous apporterons déjà les précisions suivantes.

Une distinction nette entre une erreur fortuite statistique vraie et une erreur systématique vraie, est, en pratique, impossible. On traite souvent des erreurs systématiques variables, d'erreurs fortuites.

En analyse chromatographique l'erreur systématique est fréquemment d'un ordre de grandeur bien supérieur à celui de l'erreur fortuite. Malgré cela, nous conviendrons de ce qui suit.

Les groupes de données dont il sera question plus loin sont exemptes d'erreurs systématiques. Nous appellerons «données» les résultats de mesure. 11 importe peu que les données traduisent la composition qualitative d'un échantillon, la teneur en volumes $\%$, poids $\%$ etc. ... , ou qu'elles expriment une valeur de $R_{F}$, un volume de rétention, un indice de rétention, un coefficient de diffusion ou une polarité.

Pour pouvoir porter un jugement ou prendre une décision, à partir des données, les conditions suivantes doivent être remplies:

1. Les données doivent être exemptes d'erreurs systématiques ou servir à la recherche des erreurs systématiques ou bien on doit être sûr que les erreurs systématiques n'influencent pas le jugement ou la décision.

2. Les données doivent représenter la valeur moyenne fournie par plusieures mesures; Moyenne $=\bar{x}$.

3. L'écart-type des données doit être connu; Ecarttype $=s$
4. Le nombre de mesures répétées ou comparatives, à partir desquelles la valeur moyenne a été calculée doit être connu; Nombre de mesures $=\mathrm{n}$.

Sans la connaissance des trois grandeurs caractéristiques $\bar{x}$, s et $n$, on ne peut jamais, à partir des données porter un jugement ou prendre une décision qui soient scientifiquement indiscutables.

Chaque énoncé mathématique-statistique n'offre qu'une certitude limitée que nous appellerons certitude statistique, $\mathrm{S}$, en $\%$.

On commence à admettre, au plan international, les règles suivantes:

on choisit $S=95 \%$ pour tous les contrôles de produits.

On choisit $S=99 \%$ quand des conclusions scientifiques doivent être tirées.

On choisit $\mathrm{S}=99,9 \%$ quand des décisions dépendent des investissements importants et que des valeurs matérielles élevées sont en jeu.

La précision de tous les essais et résultats statistiques dépend de la certitude statistique choisie.

\section{Valeur aberrante, Valeur moyenne, Ecart-type}

C'est le propre du «hasard» que des erreurs excessives puissent apparaître dans chaque série de mesures. Il faut au moins trois mesures répétées pour pouvoir considérer une mesure comme totalement fausse. On reconnaîtra d'autant plus sûrement la valeur aberrante que la mesure aura été répétée un plus grand nombre de fois.

Les mesures aberrantes doivent être éliminées avant que l'on puisse utiliser les données pour prendre une décision A notre avis, la meilleure méthode de repérage des valeurs aberrantes est la méthode de Nalimov. Elle présente des avantages par rapport aux nombreuses autres méthodes de détermination des valeurs aberrantes.

\section{Méthode de Nalimov}

Pour l'utiliser, on doit calculer à partir de toutes les données, y compris les valeurs aberrantes, une valeur moyenne provisoire $\overline{\mathrm{x}}$ et un écart-type provisoire $\mathrm{s}$.

1. Calcul de la valeur moyenne provisoire $\bar{x}$ à partir de toutes les valeurs élémentaires

$$
\text { Formule: } \begin{aligned}
& \bar{x}=\left(x_{1}+x_{2}+x_{3}+\ldots\right) / n \\
& n=\text { nombre total de mesures } \\
& \text { exemple: } \\
& x_{1}=30,41 \\
& x_{2}=30,05 \\
& x_{3}=30,49 \\
& x_{4}=32,06 \\
& \bar{x}=123,01 / 4 \\
& \bar{x}=30,753
\end{aligned}
$$




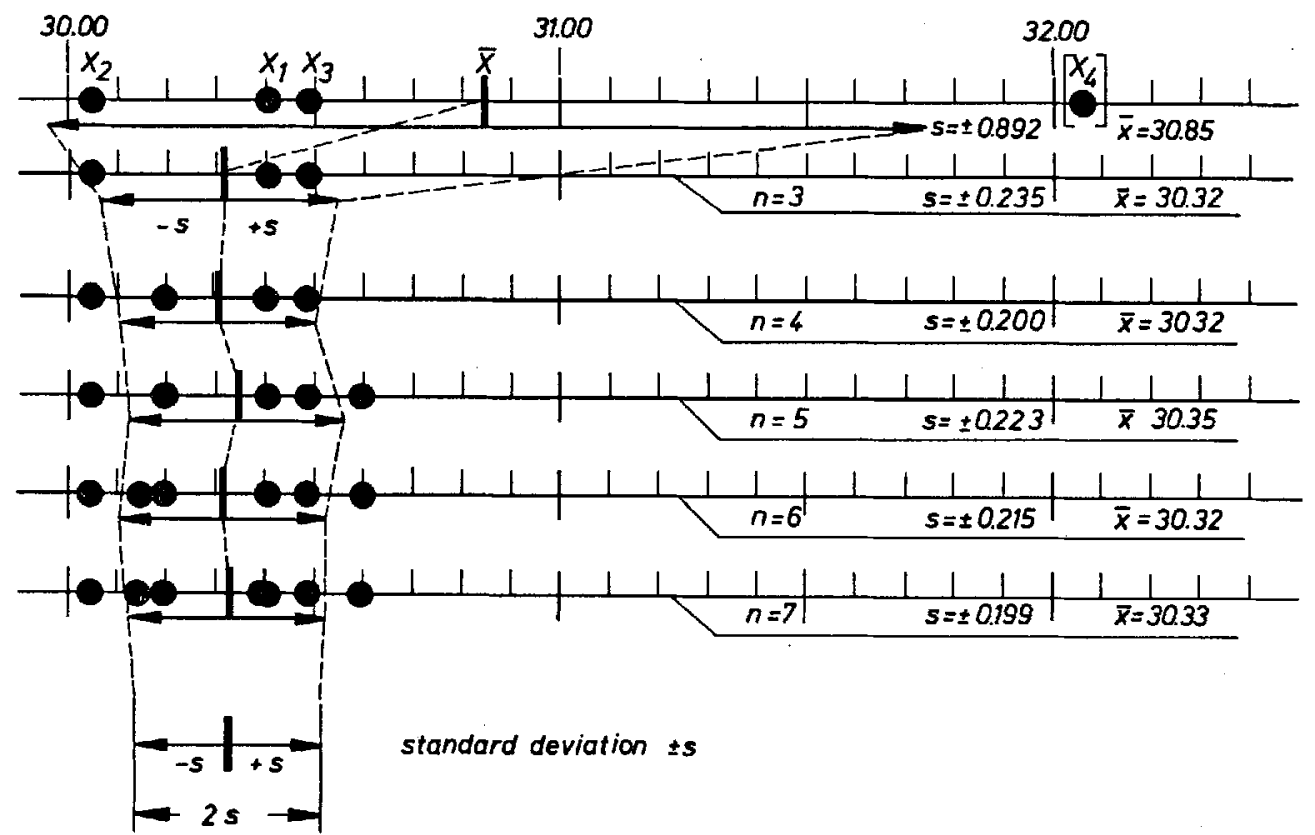

Fig 1

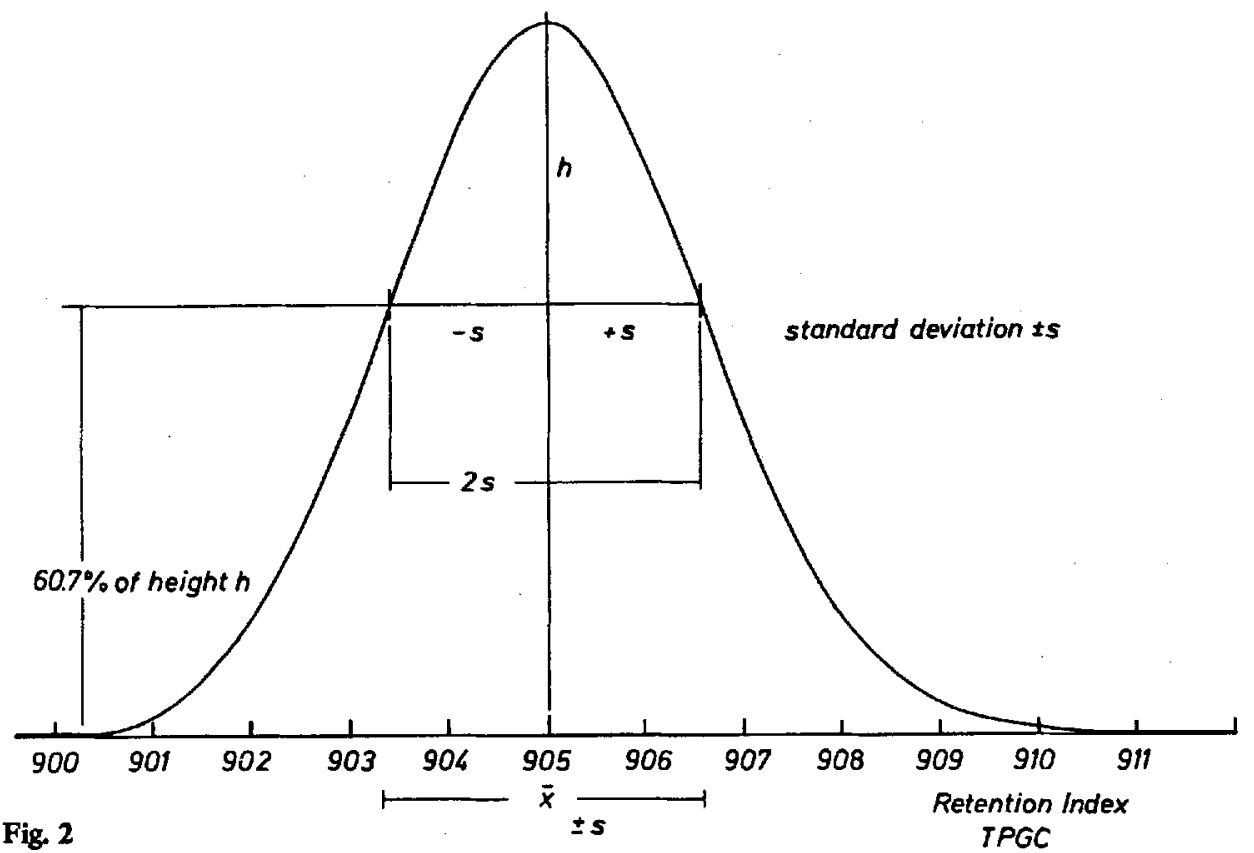

2. Calcul de l'écart-type provisoire s à partir de toutes les valeurs élémentaires

exemple:

$\mathrm{x}^{2}$

Formule: (celle-ci convient particulièrement pour l'utilisation de petites machines à calculer)

$$
\begin{aligned}
& \mathrm{x}_{1}=30,41 \\
& x_{2}=30,05 \\
& \mathrm{x}_{\mathbf{3}}=30,49 \\
& \mathrm{x}_{4}=32,06 \\
& \Sigma \mathrm{x}=123,01 \quad \Sigma \mathrm{x}^{2} 3.785 \\
& (\Sigma \mathrm{x})^{2}=15.131,4601 \\
& \begin{aligned}
s & = \pm \sqrt{\frac{1}{3}\left(3785,2543-\frac{1}{4} \cdot 15131,4601\right)} \\
& = \pm 0,8924
\end{aligned}
\end{aligned}
$$$$
s= \pm \sqrt{\frac{1}{n-1} \cdot\left[\Sigma\left(x^{2}\right)-\frac{1}{n}(\Sigma x)^{2}\right]}
$$$$
\mathrm{n}=\text { nombre total de mesures }
$$ 


\section{Remarque:}

Lorsque l'on utilise une machine à calculer on doit, dans

les résultats intermédiaires, conserver toutes les décimales. C'est seulement dans le résultat final qu'on en réduira le nombre de telle manière que son expression soit en accord avec la précision de la mesure.

\section{Mise en evidence des valeurs aberrantes}

On examine les données pour y déceler les valeurs aberrantes. La (ou les) valeur(s) la (les) plus élevée(s) et la (les) plus faible(s) peuvent être supposées aberrantes.

Eu suite on compare les valeurs aberrantes à une valeur calculée selon la formule 1 en utilisant le facteur de Nalimov dépendant de $n, N(95), N(99)$ ou $N(99,9)$, donné au tableau 1.

\section{Formule d'essais 1:}

$x^{*} \quad$ n'est pas une valeur aberrante, si: $\left|x^{*}-\bar{x}\right|$ est inférieur à $N(95) \cdot s$

$x^{*}$ est vraisemblablement une valeur aberrante, si: $\left|x^{*}-\bar{x}\right|$ est égal ou supérieur à $N(95) \cdot s$

$x^{*} \quad$ est une valeur nettement aberrante, si: $\left|x^{*}-\bar{x}\right|$ est égal ou supérieur à $N(99) \cdot s$

$x^{*} \quad$ est une valeur très fortement aberrante, si: $\left|x^{*}-\bar{x}\right|$ est égal ou supérieure à $N(99,9) \cdot s$

\section{Application}

Dans l'exemple numérique cité plus haut, il apparait: (voir tableau 1)

$$
\begin{gathered}
\mathrm{N}(95) \cdot \mathrm{s}=1,425 \cdot 0,8924=1,2717 \\
\mathrm{~N}(99) \cdot \mathrm{s}=1,485 \cdot 0,8924=1,3252 \\
\mathrm{~N}(99,9) \cdot \mathrm{s}=1,498 \cdot 0,8924=1,3368 \\
\mathrm{x}_{1}=\mathrm{x}^{*}=32,06 ;\left|\mathrm{x}^{*}-\overline{\mathrm{x}}\right|=32,06-30,753=1,3070
\end{gathered}
$$

$x_{1}, x_{2}$ et $x_{3}$ donneraient une différence beaucoup plus faible.

Il en résulte que: seule la valeur $\mathrm{x}_{4}=32,06$ est, avec une probabilité de $95 \%$ une valeur aberrante. Elle doit être éliminée.

Les données sont alors exemptes de valeurs aberrantes.
On doit ensuite calculer les valeurs caractéristiques finales $\bar{x}, s$, et $n$

$n=3$

$\bar{x}_{A}=30,317$ (parce que: $(30,41+30,05+30,49) / 3=30,317$ )

$s_{A}= \pm 0,2345$ (parce que $\sqrt{\frac{1}{2}\left(2757,4107 \frac{1}{3} \cdot 8271,9025\right)}$ $= \pm 0,2345$ )

C'est seulement alors qu'un jugement fondé sur des données mathématiques peut être porté:

Le résultat de mesure $\bar{x}_{A}$ diffêre-t-il vraiment d'un autre résultat $\bar{x}_{\mathrm{B}}$ ou d'une valeur théorique $W$ ? Du point de vue qualité de la mesure utilisée qui donne l'écart-type $s_{A}$, differe-t-il vraiment d'un autre procédé de mesure qui donne des valeurs avec un écart-type $\mathrm{s}_{\mathrm{B}}$ ?

Ces questions, ainsi qu'un essais de haute sensibilité concernant les différences des valeurs moyennes de mesures couplés et une méthode graphique simple de statistique mathématique sèront traités dans la série suivante de pages vertes.

Les méthodes de calculs traitées ici ou celles qui le seront ultérieurement, exigent que les données soient statistiquement «normalement distribuées». Les séries suivantes de pages vertes traiteront d'une méthode concemant ces questions.

Pour terminer, nous expliquerons ici la signification de la valeur moyenne $\bar{x}$ et de l'écart-type $s$ par les figures 1 et 2. La figure 1 représente une échelle de valeurs. Les quatre valeurs numériques de notre exemple de calcul y ont été reportées. On voit combien le domaine de l'écarttype dépasse les valeurs qui sont plus élevées et plus basses que la valeur moyenne.

Si on avait fait plus de quatre mesures dont on aurait encore ćliminé une autre valeur aberrante, on aurait eu une valeur moyenne différente et on aurait trouvé un écart-type légèrement plus faible.

La figure 1 indique sur des échelles de valeurs dilatées, les modifications de données auxquelles on peut s'attendre.

Sur la figure 2, la notion de valeur moyenne et d'écarttype a été appliquée au temps de rétention d'un pic de chromatographie en phase gazeuse.

Quand on calcule l'indice de rétention d'un pic la valeur obtenue devient en fait comprise dans l'écart-type qui correspond à la forme du pic. Pour cet exemple particulier, nous supposons que l'analyse a été faite à température programmée de sorte que l'échelle des indices de rétention sera linéaire. 